https://doi.org/10.36105/iut.2020n32.06

\title{
CONTEXTO GENERAL DE LA PREVENCIÓN DE LAVADO DE DINERO Y FINANCIAMIENTO AL TERRORISMO EN MÉXICO
}

\author{
GENERAL CONTEXT OF THE PREVENTION OF MONEY \\ LAUNDERING AND TERRORISM FINANCING IN MEXICO
}

\author{
María Angélica Cervantes Vera* \\ Investigadora independiente, México \\ angelika.cervantes@gmail.com
}

\section{RESUMEN:}

En la actualidad las actividades ilícitas que están vinculadas al Lavado de Dinero y Financiamiento al Terrorismo (LD/FT) a diario cobran relevancia como consecuencia de la globalización y el alcance de las comunicaciones y las nuevas tecnologías que facilitan el crecimiento y aumento de la criminalidad y los delitos asociados, esto sucede mediante los intercambios comerciales operados por los grupos delictivos que transaccionan internacionalmente de manera rápida y efectiva, dificultando el rastreo de los recursos económicos (dinero) alrededor del mundo.

Palabras clave:

Lavado de dinero, financiamiento al terrorismo, globalización, criminalidad.

\section{ABSTRACT:}

At present, the illicit activities that are linked to Money Laundering and Terrorism Financing (ML / FT) on a daily basis are gaining relevance as a result of globalization and the scope of communications and new technologies that facilitate the growth and increase of crime and associated crimes, this happens

* Maestra en Derecho. Especialista certificada en materia de Prevención de Lavado de Dinero y Financiamiento al Terrorismo por la Comisión Nacional Bancaria y de Valores. 
through commercial exchanges operated by criminal groups that transact internationally quickly and effectively, making it difficult to trace economic resources (money) around the world.

\section{Keywords:}

Money laundry, terrorism financing, globalizarion, criminality.

SUMARIO: Resumen. 1. Aspecto internacional del lavado de dinero y financiamiento al terrorismo. 2. Lavado de dinero en el ámbito de la economía nacional. 3. Las actividades vulnerables en México. 4. Conclusiones.

\section{ASPECTO INTERNACIONAL DEL LAVADO DE DINERO Y EL FINANCIAMIENTO AL TERRORISMO}

Dado que el LD/FT es un mal internacional susceptible de afectar la economía de cualquier país, se le da mucha importancia a la prevención del lavado de dinero y financiamiento al terrorismo (PLD/FT), esto es así, porque los delitos relacionados con el LD/FT se van actualizando y traspasando las fronteras y se requiere de un control exhaustivo por los países para evitar su propagación, es por ello que en 1989 se creó un organismo intergubernamental contra el blanqueo de capitales, financiamiento al terrorismo y la proliferación de armas de destrucción masiva denominado Grupo de Acción Financiera, mejor conocido como GAFI, cuyo propósito o mandato, ${ }^{1}$ en términos generales, es crear estándares y promover la efectiva implementación de medidas legales, regulatorias y operativas para combatir el lavado de activos, el financiamiento al terrorismo y la identificación de otras amenazas relacionadas con la integridad del sistema financiero internacional para evitar ser vulnerados. Dichos estándares internacionales son llamados las 40 Recomendaciones ${ }^{2}$ (las 40 Recomendaciones).

Las 40 Recomendaciones han tenido diversas modificaciones a lo largo del tiempo, la primera versión fue emitidas por GAFI en abril de 1990 (que tenían por objeto proporcionar un plan de acción necesario para la lucha contra el lavado de dinero). Las 40 Recomendaciones fueron revisadas en 1996 y reformuladas sustancialmente en 2003. En el año 2001, GAFI emitió en 20018 (ocho) Recomendaciones Especiales específicas en materia de Financiamiento al Terrorismo derivado de los sucesos ocurridos en las Torres Gemelas en Nueva York; en 2004 fueron completadas con una $9^{\mathrm{a}}$ Recomendación Especial adicional relacionada con el movimiento transfronterizo de efectivo. Posteriormente, el 16 de febrero de 2012, GAFI publicó una nueva versión de sus Recomendaciones, en las que se integran las medidas esenciales contenidas en las anteriores Cuarenta Recomendaciones, con las 9 Recomendaciones Especiales, que constituyen los estándares

1 Mandato 2012-2020, emitido por Grupo de Acción Financiera, abril 20, 2020, en Washington D.C., obtenido de la página de internet: https://www.cnbv.gob.mx/PrevencionDeLavadoDeDinero/Documents/Mandato GAFI_2012-2020\%20ESP\%20versi\%C3\%B3n\%20final\%20mayo11.pdf

2 Estándares internacionales sobre la lucha contra el lavado de activos y el financiamiento del terrorismo y la proliferación. Las Recomendaciones de GAFI. Febrero de 2012, obtenido de la página de internet: https:// www.fatf-gafi.org/media/fatf/documents/recommendations/pdfs/FATF-40-Rec-2012-Spanish.pdf 
internacionales utilizados por más de 180 países para combatir eficazmente y de manera global los mencionados delitos, así como para evitar el uso de los sistemas financieros con fines ilícitos. ${ }^{3}$

El lavado de dinero es considerado, según José de Jesús González Rodríguez, en su libro "El lavado de dinero en México, escenarios, marco legal y propuestas legislativas", ${ }^{4}$ como uno de los delitos más graves de la criminalidad organizada contemporánea. Su evolución en el derecho internacional y en los marcos legales de los estados, demuestra con suficiencia que se trata de una práctica que ha marcado sus propias tendencias en la sociedad actual.

Las actividades criminales relacionadas con el LD/FT no solo lastiman principalmente la economía nacional, sino también generan riesgos de debilidad o inestabilidad de los mercados financieros internacionales y por consecuencia, la disminución o falta de control de las políticas económicas, afectan adversamente las monedas, las tasas de interés, propicia baja recaudación fiscal y pérdida de ingresos fiscales nacionales, erosiona en mayor medida la economía de los países, principalmente los que se encuentran en proceso de desarrollo, colocándose éstos, en un blanco fácil para la realización de operaciones con recursos de procedencia ilícita por la delincuencia organizada.

Los delitos de venta ilegal de armas, el tráfico de drogas, el contrabando, y otras actividades del crimen organizado asociadas al LD/FT pueden producir grandes ganancias a sus perpetradores. Pero no son los únicos delitos, existen otros como el robo, el fraude, el soborno y tantas nuevas operaciones realizadas a través de elaboradas estructuras jurídicas en las que se involucran nuevas tecnologías, así como el llamado, fraude electrónico que también producen buenas ganancias y grandes incentivos para los delincuentes de cuello blanco, toda vez que legitiman de manera avanzada, a la luz de esas estructuras jurídicas y de nuevas tecnologías, los fondos obtenidos ilícitamente a través del LD/FT.

El LD/FT también generan otro tipo de riesgos sociales y de seguridad, potencialmente devastadores, que pueden ocurrirles a cualquier país afectando principalmente a los que se encuentran en vías de desarrollo o con economías emergentes y/o países con sistemas de prevención de lavado de dinero frágiles, produciendo consecuencias económicas por el aumento al gasto público para invertir en mayores recursos en la administración de justicia y sus colaterales (trasladando o redirigiendo el gasto público para combatir el narcotráfico, narcomenudeo, contrabando, y a los delincuentes que participan en estas actividades ilícitas), como puede ser incluso, mayor gasto para el sistema de salud pública por la prevención en campañas de prevención de drogas o su combate.

3 Obtenido del artículo publicado por la Comisión Nacional Bancaria y de Valores en la siguiente liga: https:// www.gob.mx/cms/uploads/attachment/file/80948/VSPP_GAFI_13042016.pdf

4 José de Jesús González Rodríguez. "El lavado de dinero en México, escenarios, marco legal y propuestas legislativas". Documento de Trabajo número 66, abril de 2009. Centro de Estudios Sociales y de Opinión Publica. 


\section{LAVADO DE DINERO EN EL ÁMBITO DE LA ECONOMÍA NACIONAL}

Volviendo a la globalización, las operaciones con recursos de procedencia ilícita no son específicos de un país, todos los países están expuestos y pueden ser utilizados por estar geo ubicados en una posición que permite que los delitos relacionados se realicen en mayor o menor medida dependiendo de lo estricto y coercitivo de sus legislaciones.

La PLD/FT se da en dos ámbitos muy importantes de la economía, por un lado tenemos la que regula al sector financiero y a las instituciones financieras internacionales o nacionales, que de conformidad con las recomendaciones de GAFI están obligados a emitir e implementar medidas y procedimientos para prevenir el lavado de dinero y el financiamiento al terrorismo y evitar ser el medio para que los delincuentes coloquen recursos de procedencia ilícita en sus instituciones financieras. Por otro lado tenemos la PLD/FT que se da en el sector empresarial, actividades llamadas formalmente por GAFI en sus recomendaciones 22 y 23 como Actividades Profesionales No Financieras Designadas (APNFD); las APNFD's son seleccionadas por cada país de acuerdo a sus niveles de riesgo y el grado de exposición a que están sujetas y dependiendo de sus tendencias de operatividad con recursos de procedencia ilícita, que en términos generales contemplan, entre otros, a los abogados, contadores, fideicomisos empresariales, fedatarios públicos, prestamistas, arrendadores, etcétera.

México no es la excepción, y los delitos relacionados con lavado de dinero cobran cada vez más fuerza, particularmente el crimen organizado que como bien sabemos, es un negocio muy rentable puesto que las actividades u operaciones que se involucran generan ganancias millonarias, mismas que se tratan de incorporar en el sistema financiero mexicano como operaciones ordinarias, lícitas, afectando la economía nacional, utilizando diversos canales de transmisión, sectores de la economía, territorios y una gama de productos o servicios que les facilita el ingreso de recursos ilícitos.

Por lo anterior, el delito de lavado de dinero se incorporó inicialmente al ámbito legal mexicano con la inclusión del artículo 115 Bis del Código Fiscal de la Federación. Mas tarde, en mayo de 1996 entró en vigor el Decreto que reformó diversos artículos del Código Penal, del Código Fiscal de la Federación y del Código Federal de Procedimientos Penales y como consecuencia, se derogó el entonces artículo 115 Bis del Código Fiscal de la Federación, para emitirse en su lugar, el artículo 400 Bis del Código Penal Federal, vigente hasta la fecha. Es decir, el delito de lavado de dinero dejó de ser considerado un ilícito eminentemente fiscal y pasó a formar parte de la legislación punitiva mexicana.

En México la PLD/FT se implementó inicialmente de manera efectiva y eficiente en las instituciones integrantes del Sistema Financiero Mexicano, bajo la supervisión y vigilancia de la Comisión Nacional Bancaria y de Valores (CNBV); cada tipo de Institución Financiera cuenta con disposiciones de carácter general que regulan la PLD/FT. Las instituciones que conforman el sistema financiero 
son, entre otras, las Instituciones de Crédito; Almacenes Generales de Depósito; Casas de Cambio; Sociedades Financieras de Objeto Múltiple; Uniones de Crédito; Sociedades Financieras Populares; Sociedades Cooperativas de Ahorro y Préstamo; Casas de Bolsa; Fondos de Inversión, etcétera.

En el ámbito nacional, la Ley Federal para la Prevención e Identificación de Operaciones con Recursos de Procedencia Ilícita (LFPIORPI) regula a las APNFD, denominadas en la legislación mexicana como Actividades Vulnerables.

La LFPIORPI distingue las Actividades Vulnerables, dentro de los cuales quedan comprendidas cierto tipo de negocios y profesiones legitimas que por sus características propias pueden servir a la delincuencia organizada como medio de acceso de los recursos ilícitos a la economía formal, con la finalidad de ocultar su procedencia delictiva.

No perdiendo de vista lo anterior, a los sujetos obligados a la LFPIORPI se les requiere responsabilidad social y legal de actuar como coadyuvantes con las autoridades encargadas de la prevención del lavado de dinero para evitar llevar a cabo operaciones con recursos de procedencia ilícita y financiamiento al terrorismo o participar en las mismas.

Dentro de las Actividades Vulnerables se encuentran, entre otras, la fe pública (notarios y corredores públicos), prestamistas no financieros, los vendedores de joyas, piedras preciosas y obras de arte, juegos y sorteos (casinos), las operaciones con cheques de viajero, la compraventa de autos nuevos o usados, la donaciones recibidas por organizaciones sin fines de lucro, los arrendadores, abogados y contadores en supuestos muy concretos, los desarrolladores de bienes inmuebles, intermediarios en la venta de bienes inmuebles y los vendedores de los mismos, diversas actividades de comercio exterior, blindadores de autos, etcétera.

\section{LAS ACTIVIDADES VULNERABLES EN MÉXICO}

México forma parte de GAFI como miembro de pleno derecho y por lo tanto la implementación de las 40 Recomendaciones es obligatoria en su legislación nacional; por su parte GAFI posee mecanismos de evaluación para verificar el grado de cumplimiento de las 40 Recomendaciones, los mecanismos son dos: (i) ejercicios de autoevaluación y (ii) las evaluaciones mutuas (Evaluaciones Mutuas).

Las Evaluaciones Mutuas constituyen el proceso mediante el cual el GAFI examina el nivel de cumplimiento y nivel de efectividad de los criterios y recomendaciones que formula en materia de prevención y combate del lavado de dinero y del financiamiento al terrorismo. Todos los países que integran el organismo son sometidos a este proceso de evaluación, el cual culmina en un documento (Reporte) que da cuenta de la situación general de cada país evaluado. ${ }^{5}$

Para llevar a cabo las Evaluaciones Mutuas, los evaluadores toman en consideración "La Metodología para evaluar el cumplimiento técnico con las

5 Obtenido del artículo publicado por la Comisión Nacional Bancaria y de Valores en la siguiente liga: https:// www.gob.mx/cms/uploads/attachment/file/80948/VSPP_GAFI 13042016.pdf. 
recomendaciones del GAFI y la efectividad de los sistemas ALA/CFT", elaborado por GAFI en el año $2013^{6}$ y los factores más importantes a considerar para la realización de la evaluación serán los riesgos en materia de $\mathrm{LD} / \mathrm{FT}$ y el contexto del país en un sentido amplio y los elementos que contribuyen a ello como son:
a) La naturaleza y el alcance del lavado de activos y el financiamiento del terrorismo;
b) Las circunstancias del país, que afectan a la materialidad de las distintas Recomendaciones (por ejemplo, la composición de su economía y de su sector financiero);
c) Los elementos estructurales que sustentan el sistema ALA/CFT, y
d) Los factores contextuales que pueden influir la manera de implementa- ción de las medidas ALA/CFT y su efectividad.

Se han realizado diversas Evaluaciones Mutuas a México, respecto de las cuales, en el informe de evaluación mutua emitido por GAFI en el año 2008 (IEM 2008) determinó incumplimientos por parte del gobierno de México a sus 40 recomendaciones en materia de prevención de lavado de dinero y sus 9 recomendaciones especiales para combatir el financiamiento al terrorismo, indicando entre otros puntos lo siguiente:

“(...) las leyes que penalizan el lavado de dinero y el financiamiento al terrorismo no responden plenamente a las normas internacionales, y no hay margen para mejorar significativamente su aplicación."

"No hay medidas legales o reglamentarias de prevención del lavado de dinero y el financiamiento al terrorismo, ni de supervisión, para cualquiera de las categorías de empresas y profesiones no financieras designadas por el GAFI (desígnate nonfinancial businesses and professions DNFBPs por sus siglas en inglés), a excepción de los servicios de confianza que por ley sólo pueden ser prestados por las instituciones financieras autorizadas."

"La falta de medidas en relación con las demás categorías de DNFBPs representa una laguna importante del régimen de prevención del Lavado de Dinero y el Financiamiento al Terrorismo. Además, ninguna revisión se ha realizado a las entidades y organizaciones sin fines de lucro (NPO), así como para apoyar a la adopción de medidas para impedir el uso ilegal de las personas morales en relación con lavado de dinero y el financiamiento al terrorismo".?

Por consecuencia, el 17 de octubre de 2012 el Congreso General de los Estados Unidos Mexicanos decretó y publicó en el Diario Oficial de la Federación la Ley Federal para la Prevención e Identificación de Operaciones con Recursos de Procedencia Ilícita para darle cumplimiento a lo requerido por GAFI en el IEM 2008.

\footnotetext{
6 Obtenido de la página de internet: https://www.pld.hacienda.gob.mx/work/models/PLD/documentos/metodologia_gafi.pdf

7 Punto II de la Exposición de Motivos de la Ley Federal para la Prevención e Identificación de Operaciones con Recursos de Procedencia Ilícita, página 24, decreto publicado el 17 de octubre de 2012 por el Congreso General de los Estados Unidos Mexicanos.
} 
No ha sido fácil para las Actividades Vulnerables la interpretación y aplicación de la LFPIORPI, sus reglamento y reglas de carácter general, incluso hay diversos sectores en incumplimiento; sin embargo, existen algunos proyectos de reformas a la LFPIORPI que ahora mismo se encuentran en revisión para su publicación y entrada en vigor, lo que permitirán una mejor claridad de las obligaciones y el incremento de otras tantas, para fortalecer el régimen de prevención en estas APNDF y el mejor entendimiento de los riesgos asociados al LD/FT, tomando en cuenta en todo momento, los requerimientos hechos por GAFI en el resultado de la Evaluación Mutua realizada a México en 2017 cuyo informe fue emitido en 2018 (IEM, 2018).

La implementación de las obligaciones en materia de Prevención de Lavado de Dinero para los sujetos obligados a la LFPIORPI ha generado retos muy importantes para las autoridades reguladoras y supervisoras, porque la cultura de prevención no era considerada como una línea de negocio para las actividades profesionales y empresariales designadas puesto que es evidente que su interés se constriñía a la comercialización de sus productos o servicios y requirió de una nueva mentalidad para incorporar en sus estructuras operativas los lineamientos de las disposiciones de la materia, aún frente a la generación de posibles multas por falta de cumplimiento de las mismas.

A pesar de los años que han transcurrido desde la entrega en vigor de la LFPIORPI en el mes de julio de 2013 a la fecha, las autoridades han implementado diversos programas para que los sujetos obligados se den de alta en el padrón de Actividades Vulnerables e inicien con el cumplimiento de sus obligaciones.

¿Cuáles son las obligaciones a que se refiere la LFPIORPI?, el artículo 18 nos enuncia las siguientes:

“ARTículo 18. Quienes realicen las Actividades Vulnerables a que se refiere el artículo anterior tendrán las obligaciones siguientes:

I. Identificar a los clientes y usuarios con quienes realicen las propias actividades sujetas a supervisión y verificar su identidad basándose en credenciales o documentación oficial, así como recabar copia de la documentación;

II. Para los casos en que se establezca una relación de negocios, se solicitará al cliente o usuario la información sobre su actividad u ocupación, basándose entre otros, en los avisos de inscripción y actualización de actividades presentados para efectos del Registro Federal de Contribuyentes;

III. Solicitar al cliente o usuario que participe en Actividades Vulnerables información acerca de si tiene conocimiento de la existencia del dueño beneficiario $y$, en su caso, exhiban documentación oficial que permita identificarlo, si esta obrare en su poder; en caso contrario, declarará que no cuenta con ella;

IV. Custodiar, proteger, resguardar y evitar la destrucción u ocultamiento de la información y documentación que sirva de soporte a la Actividad Vulnerable, así como la que identifique a sus clientes o usuarios.

La información y documentación a que se refiere el párrafo anterior deberá conservarse de manera física o electrónica, por un plazo de cinco años contado a partir de la fecha de la realización de la Actividad Vulnerable, salvo que las leyes de la materia de las entidades federativas establezcan un plazo diferente; 
V. Brindar las facilidades necesarias para que se lleven a cabo las visitas de verificación en los términos de esta Ley, y

VI. Presentar los Avisos en la secretaria en los tiempos y bajo la forma prevista en esta Ley."

En seguida una breve explicación de cada una de las mencionadas obligaciones.

En relación al inciso I. Identificar significa armar un expediente único de los clientes o usuarios con los que las Actividades Vulnerables realicen operaciones, dichos expedientes estarán integrados de conformidad con lo que indican las Reglas de Carácter General (RCG) en los anexos de las mismas por tipo de cliente o usuario.

El inciso II. nos indica que las Actividades Vulnerables deberán solicitar a los clientes o usuarios con los que realicen operaciones, que acrediten de manera fehaciente a que se dedican éstos, basándose en los documentos fiscales con los que estén dados de alta en el Registro Federal de Contribuyentes (RFC), esto implica que los clientes con quienes realicen de manera reiteradas operaciones, deberán estar inscritos en el RFC.

Si bien es cierto, esto pudiera implicar consecuencias fiscales para los clientes, es preciso aclarar que la naturaleza jurídica de este ordenamiento legal es administrativa y por tanto se rige de manera supletoria por la Ley Federal del Procedimiento Administrativo.

Relativo al inciso III, nos indica que las Actividades Vulnerables deberán preguntar a los clientes o usuarios información relativa al dueño beneficiario o beneficiario final (según lo define la fracción IV del artículo 3 de las RCG) ${ }^{8}$ y en caso de que sea afirmativa la respuesta, identificar a dicho dueño beneficiario y dejar constancia. Este inciso, ha sido motivo de diversos criterios interpretativos por parte de la Unidad de Inteligencia Financiera par falta de clarificar y saber de manera precisa a quien debe considerarse como dueño beneficiario o beneficiario controlador.

8 Fracción IV, del artículo 3 de las Reglas de Carácter General de la Ley Federal para la Prevención e Identificación de Operaciones con Recursos de Procedencia Ilícita que indica lo siguiente:

$\ldots$

IV. Beneficiario Controlador, a la persona o grupo de personas que:

a) Por medio de otra o de cualquier acto, obtiene el beneficio derivado de estos y es quien, en última instancia, ejerce los derechos de uso, goce, disfrute, aprovechamiento o disposición de un bien o servicio, o

b) Ejerce el control de aquella persona moral que, en su carácter de Cliente o Usuario, lleve a cabo actos u operaciones con quien realice una Actividad Vulnerable, así como las personas por cuenta de quienes celebra alguno de ellos.

Se entiende que una persona o grupo de personas controla a una persona moral cuando, a través de la titularidad de valores, por contrato o cualquier otro acto, puede:

i) Imponer, directa o indirectamente, decisiones en las asambleas generales de accionistas, socios u órganos equivalentes, o nombrar o destituir a la mayoría de los consejeros, administradores o sus equivalentes;

ii) Mantener la titularidad de los derechos que permitan, directa o indirectamente, ejercer el voto respecto de más del cincuenta por ciento del capital social, o

iii) Dirigir, directa o indirectamente, la administración, la estrategia o las principales políticas de la misma.

No se considerarán como Beneficiarios Controladores a los poderdantes o mandantes en los casos en que sus apoderados o mandatarios celebren el acto u operación de que se trate; (Párrafo adicionado D.O.F. 24 julio 2014) 
Concretamente se busca que las Actividades Vulnerables cuestionen a los clientes para conocer si detrás de la operación que se está realizando, existe un tercero que sin ser parte de la misma es quien detentará los derechos y quien en últimas gozará y disfrutará el producto de la operación realizada.

El inciso IV implica que los sujetos obligados deberán conservar los expedientes de sus clientes o usuarios durante el transcurso de cinco años y por lo tanto deberán asegurarse de contar con las medidas de seguridad y resguardo que evite su destrucción o pérdida.

Los sujetos obligados son susceptibles de recibir visitas de verificación por parte del área encargada de la verificación dependiente del Servicio de Administración Tributaria (SAT), de conformidad con lo que dispone la fracción V de este artículo 18 de la LFPIORPI para determinar el grado de cumplimiento de las obligaciones de ésta, por tanto, deberán poner a disposición de la mencionada autoridad, los documentos que le requieran en la realización de las visitas de verificación.

Se aclara que evidentemente para llevar a cabo estas visitas, el verificador deberá acreditar su personalidad y presentar el oficio correspondiente emitido por la autoridad competente que funde y motive la visita en cuestión, su ámbito de verificación comprenderá únicamente operaciones realizadas por las Actividades Vulnerables en los cinco años previos a la realización de la visita.

Y por último el artículo 18 fracción VI indica que los sujetos obligados deberán presentar Avisos en el portal del SAT de prevención de lavado de dinero, previa alta de los sujetos considerados como Actividades Vulnerables, en la siguiente liga de internet: https://sppld.sat.gob.mx/pld/interiores/sppld.html. Dichos Avisos contemplarán la información relativa a la operación realizada por los clientes o usuarios, esto es, datos generales de quien realiza la operación, tipo de operación celebrada, monto de operación, forma de pago de la misma, en su caso información de dueño beneficiario o beneficiario final, entre otra.

Estas son obligaciones muy importantes pero no las únicas y uno de los mayores propósitos de las 40 Recomendaciones, la LFPIORPI y las reformas a dicho ordenamiento, es que los sujetos obligados conozcan de manera clara y precisa los riesgos asociados en materia de LD/FT y no solo se cumpla para evitar multas porque la vulnerabilidad de estas actividades se determinó por ser utilizados con mayor frecuencia en operaciones con recursos de procedencia ilícita o bien porque derivado de su actividad cotidiana se puede obtener información relativa a esas operaciones

En un principio, las Actividades Vulnerables veían como una carga adicional a las propias obligaciones fiscales, el cumplimiento de la LFPIORPI, sin embargo, aquellas que lo han implementado en sus procesos operativos lo han hecho como medidas de control interno efectivo para saber con quienes realizan operaciones, ya sea de manera directa o en su calidad de intermediarios, como lo es el fedatario público.

Dentro de las mencionadas obligaciones, existen dos que dispone el artículo 17 de la Ley que son la identificación de los clientes o usuarios con quienes 
las Actividades Vulnerables realizan operaciones y la presentación de los Avisos. Para tales fines, la Ley determina dos umbrales: a) Umbral de Identificación y b) Umbral de Avisos. Estos umbrales a partir del $1^{\circ}$ de febrero de 2016 estarán calculados en Unidades de Medidas y Actualización (UMA o UMA's) de acuerdo al valor asignado por el Instituto Nacional de Estadística y Geografía que publique en el Diario Oficial de la Federación; ${ }^{9}$ estos umbrales son por el valor de la operación que será realizada, ya sea en lo individual o por suma acumulada de varias operaciones en un transcurso de seis meses.

Los Umbrales de Identificación concretamente obligan a los sujetos obligados a crear un expediente único de los clientes o usuarios con los que se realizan dichas Actividades Vulnerables, dependiendo del tipo de cliente de que se trate, de acuerdo a lo que distingue el artículo 12 de las reglas de carácter general de la Ley (RGC), tomando en consideración también, el valor de la operación en UMA's.

Hay operaciones que siempre serán sujetas de identificación y creación de expediente único, independientemente del monto de la operación.

Los Umbrales de Aviso son los montos en UMA's que deben de considerarse para la presentación de los Avisos por las operaciones celebradas por montos iguales o superiores a los valores determinados para cada actividad vulnerable.

De la misma forma que los Umbrales de Identificación, hay operaciones que por el simple hecho de realizarse, independiente del valor de la misma, será sujeta de presentación de Aviso.

A continuación una tabla que muestra cada Actividad Vulnerable, en términos del artículo 17 de la LFPIORPI y los Umbrales de Identificación y Umbrales de Aviso, con valores en UMA's para que se puedan distinguir claramente:

\begin{tabular}{|c|l|c|c|}
\hline $\begin{array}{c}\text { FRACCIÓN DEL } \\
\text { ARTÍCULO 17 } \\
\text { DE LA LEY }\end{array}$ & \multicolumn{1}{|c|}{ ACTIVIDAD VULNERABLE } & $\begin{array}{c}\text { UMBRAL DE } \\
\text { IDENTIFICA- } \\
\text { CIÓN EN UMAS }\end{array}$ & $\begin{array}{c}\text { UMBRAL DE } \\
\text { AVISO EN } \\
\text { UMAS }\end{array}$ \\
\hline \multirow{2}{*}{ F.I. } & $\begin{array}{c}\text { PRÁCTICA DE JUEGOS Y APUESTAS, CONCURSOS O SORTEOS } \\
\text { QUE REALICEN ORGANISMOS DESCENTRALIZADOS, O SE LLE- } \\
\text { VEN A CABO BAJO LA LEY DE JUEGOS Y SORTEOS, EN LOS } \\
\text { SIGUIENTES TÉRMINOS: }\end{array}$ & \\
\cline { 2 - 4 } & $\begin{array}{l}\text { BOLETOS, FICHAS, O CUAL- } \\
\text { QUIER SIMILAR A LA PRÁCTI- } \\
\text { CA, Y EL PAGO DEL VALOR DE } \\
\text { LOS MISMOS. }\end{array}$ & \multicolumn{2}{|c}{$\mathbf{3 2 5}$} \\
\cline { 2 - 4 } & $\begin{array}{l}\text { PAGO DE PREMIOS O SORTEOS } \\
\text { EN GENERAL. }\end{array}$ & $\mathbf{3 2 5}$ & 645 \\
\hline
\end{tabular}

9 La Unidad de Medida y Actualización (UMA) es la referencia económica en pesos para determinar la cuantía del pago de las obligaciones y supuestos previstos en las leyes federales, de las entidades federativas, así como en las disposiciones jurídicas que emanen de todas las anteriores. Obtenido de la página de internet del INEGI, en la siguiente liga: https://www.inegi.org.mx/temas/uma/\#: :text=La\%20Unidad\%20de\%20Medida $\% 20 \mathrm{y}$, emanen $\% 20 \mathrm{de} \% 20$ todas $\% 201 \mathrm{las} \% 20$ anteriores. 


\begin{tabular}{|c|c|c|c|}
\hline $\begin{array}{l}\text { FRACCIÓN DEL } \\
\text { ARTÍCULO } 17 \\
\text { DE LA LEY }\end{array}$ & ACTIVIDAD VULNERABLE & $\begin{array}{l}\text { UMBRAL DE } \\
\text { IDENTIFICA- } \\
\text { CIÓN EN UMAS }\end{array}$ & $\begin{array}{l}\text { UMBRAL DE } \\
\text { AVISO EN } \\
\text { UMAS }\end{array}$ \\
\hline \multirow[t]{2}{*}{ F.II. } & $\begin{array}{l}\text { TARJETAS DE SERVICIOS, DE } \\
\text { CRÉDITO, DE TARJETAS PREPA- } \\
\text { GADAS Y DE TODAS AQUE- } \\
\text { LLAS QUE CONSTITUYAN INS- } \\
\text { T R U M E N T O S D E } \\
\text { ALMACENAMIENTO DE VALOR } \\
\text { MONETARIO, QUE NO SEAN } \\
\text { EMITIDAS O COMERCIALIZA- } \\
\text { DAS POR ENTIDADES FINAN- } \\
\text { CIERAS. SIEMPRE Y CUANDO, } \\
\text { EN FUNCIÓN DE TALES ACTIVI- } \\
\text { DADES: EL EMISOR O COMER- } \\
\text { CIANTE DE DICHOS INSTRU- } \\
\text { MENTOS MANTENGA UNA } \\
\text { RELACIÓN DE NEGOCIOS CON } \\
\text { EL ADQUIRENTE; DICHOS INS- } \\
\text { TRUMENTOS PERMITAN LA } \\
\text { TRANSFERENCIA DE FONDOS, } \\
\text { O SU COMERCIALIZACIÓN SE } \\
\text { HAGA DE MANERA OCASIO- } \\
\text { NAL. EN EL CASO DE TARJETAS } \\
\text { DE SERVICIOS O DE CRÉDITO, } \\
\text { CUANDO EL GASTO MENSUAL } \\
\text { ACUMULADO EN LA CUENTA } \\
\text { DE LA TARJETA SEA IGUAL O } \\
\text { SUPERIOR AL EQUIVALENTE A } \\
\text { OCHOCIENTAS CINCO VECES } \\
\text { EL SALARIO MÍNIMO VIGENTE } \\
\text { EN EL DISTRITO FEDERAL. }\end{array}$ & 825 & 1,285 \\
\hline & TARJETAS PREPAGADAS & 645 & 645 \\
\hline F.III & $\begin{array}{l}\text { L A E M I S I Ó N Y Y } \\
\text { COMERCIALIZACIÓN HABI- } \\
\text { TUAL O PROFESIONAL DE CHE- } \\
\text { QUES DE VIAJERO, DISTINTA A } \\
\text { LA REALIZADA POR LAS ENTI- } \\
\text { DADES FINANCIERAS. }\end{array}$ & SIEMPRE & 645 \\
\hline F.IV & $\begin{array}{l}\text { EL OFRECIMIENTO HABITUAL } \\
\text { O PROFESIONAL DE OPERACIO- } \\
\text { NES DE MUTUO O DE } \\
\text { GARANTIIA O DE OTORGA- } \\
\text { MIENTO DE PRÉSTAMOS O } \\
\text { CRÉDITOS, CON O SIN } \\
\text { GARANTÍA, POR PARTE DE SU- } \\
\text { JETOS DISTINTOS A LAS ENTI- } \\
\text { DADES FINANCIERAS. }\end{array}$ & SIEMPRE & 1,605 \\
\hline
\end{tabular}




\begin{tabular}{|c|c|c|c|}
\hline $\begin{array}{l}\text { FRACCIÓN DEL } \\
\text { ARTÍCULO } 17 \\
\text { DE LA LEY }\end{array}$ & ACTIVIDAD VULNERABLE & $\begin{array}{l}\text { UMBRAL DE } \\
\text { IDENTIFICA- } \\
\text { CIÓN EN UMAS }\end{array}$ & $\begin{array}{l}\text { UMBRAL DE } \\
\text { AVISO EN } \\
\text { UMAS }\end{array}$ \\
\hline \multirow[b]{3}{*}{ F.V } & $\begin{array}{l}\text { SERVICIOS DE CONSTRUCCIÓN } \\
\text { O DESARROLLO DE BIENES IN- } \\
\text { MUEBLES. } \\
\end{array}$ & SIEMPRE & 8,025 \\
\hline & $\begin{array}{l}\text { INTERMEDIACIÓN EN LA } \\
\text { TRANSMISIÓN DE LA PROPIE- } \\
\text { DAD. }\end{array}$ & SIEMPRE & 8,025 \\
\hline & $\begin{array}{l}\text { CONSTITUCIÓN DE DERECHOS } \\
\text { SOBRE DICHOS BIENES, EN LOS } \\
\text { QUE SE INVOLUCREN OPERA- } \\
\text { CIONES DE COMPRA O VENTA } \\
\text { DE LOS PROPIOS BIENES POR } \\
\text { CUENTA O A FAVOR DE CLIEN- } \\
\text { TES DE QUIENES PRESTEN DI- } \\
\text { CHOS SERVICIOS. } \\
\end{array}$ & SIEMPRE & 8,025 \\
\hline F.VI. & $\begin{array}{l}\text { LA COMERCIALIZACIÓN O } \\
\text { INTERMEDIACIÓN HABITUAL } \\
\text { O PROFESIONAL DE METALES } \\
\text { PRECIOSOS, PIEDRAS PRECIO- } \\
\text { SAS, JOYAS O RELOJES, EN LAS } \\
\text { QUE SE INVOLUCREN OPERA- } \\
\text { CIONES DE COMPRA O VENTA } \\
\text { DE DICHOS BIENES. } \\
\end{array}$ & 805 & 1,605 \\
\hline F.VII. & $\begin{array}{l}\text { SUBASTAS O COMERCIALIZA- } \\
\text { CIÓN DE OBRAS DE ARTE. }\end{array}$ & 2,410 & 4,815 \\
\hline F.VIII. & $\begin{array}{l}\text { LA COMERCIALIZACIÓN O } \\
\text { DISTRIBUCIÓN HABITUAL } \\
\text { PROFESIONAL DE VEHÍCULOS, } \\
\text { NUEVOS O USADOS, YA SEAN } \\
\text { AÉREOS, MARÍTIMOS O TE- } \\
\text { RRESTRES. }\end{array}$ & 3,210 & 6,420 \\
\hline F. IX & $\begin{array}{l}\text { LA PRESTACIÓN HABITUAL O } \\
\text { PROFESIONAL DE SERVICIOS } \\
\text { DE BLINDAJE DE VEHÍCULOS } \\
\text { TERRESTRES, NUEVOS O USA- } \\
\text { DOS, ASÍ COMO DE BIENES IN- } \\
\text { MUEBLES. }\end{array}$ & 2,410 & 4,815 \\
\hline F. $X$. & $\begin{array}{l}\text { LA PRESTACIÓN HABITUAL O } \\
\text { PROFESIONAL DE SERVICIOS } \\
\text { DE TRASLADO O CUSTODIA DE } \\
\text { DINERO O VALORES. }\end{array}$ & SIEMPRE & 3,210 \\
\hline
\end{tabular}




\begin{tabular}{|c|c|c|c|}
\hline $\begin{array}{l}\text { FRACCIÓN DEL } \\
\text { ARTÍCULO } 17 \\
\text { DE LA LEY }\end{array}$ & ACTIVIDAD VULNERABLE & $\begin{array}{l}\text { UMBRAL DE } \\
\text { IDENTIFICA- } \\
\text { CIÓN EN UMAS }\end{array}$ & $\begin{array}{l}\text { UMBRAL DE } \\
\text { AVISO EN } \\
\text { UMAS }\end{array}$ \\
\hline \multirow{6}{*}{ F. XI } & \multicolumn{3}{|c|}{$\begin{array}{l}\text { LA PRESTACIÓN DE SERVICIOS PROFESIONALES, DE MANERA } \\
\text { INDEPENDIENTE, SIN QUE MEDIE RELACIÓN LABORAL CON } \\
\text { EL CLIENTE RESPECTIVO, EN AQUELLOS CASOS EN LOS QUE } \\
\text { SE PREPARE PARA UN CLIENTE O SE LLEVEN A CABO EN NOM- } \\
\text { BRE Y REPRESENTACIÓN DEL CLIENTE CUALQUIERA DE LAS } \\
\text { SIGUIENTES OPERACIONES: }\end{array}$} \\
\hline & $\begin{array}{l}\text { A) LA COMPRAVENTA DE BIE- } \\
\text { NES INMUEBLES O LA CESIÓN } \\
\text { DE DERECHOS SOBRE ESTOS; }\end{array}$ & SIEMPRE & $\begin{array}{l}\text { CUANDO REA- } \\
\text { LICE OPERA- } \\
\text { CIONES A } \\
\text { NOMBRE DEL } \\
\text { CLIENTE }\end{array}$ \\
\hline & $\begin{array}{l}\text { B) LA ADMINISTRACIÓN Y MA- } \\
\text { NEJO DE RECURSOS, VALORES } \\
\text { O CUALQUIER OTRO ACTIVO } \\
\text { DE SUS CLIENTES; }\end{array}$ & SIEMPRE & $\begin{array}{l}\text { CUANDO REA- } \\
\text { LICE OPERA- } \\
\text { CIONES A } \\
\text { NOMBRE DEL } \\
\text { CLIENTE }\end{array}$ \\
\hline & $\begin{array}{l}\text { C) EL MANEJO DE CUENTAS } \\
\text { BANCARIAS, DE AHORRO O DE } \\
\text { VALORES; }\end{array}$ & SIEMPRE & $\begin{array}{c}\text { CUANDO REA- } \\
\text { LICE OPERA- } \\
\text { CIONES A } \\
\text { NOMBRE DEL } \\
\text { CLIENTE }\end{array}$ \\
\hline & $\begin{array}{l}\text { D) LA ORGANIZACIÓN DE } \\
\text { APORTACIONES DE CAPITAL O } \\
\text { CUALQUIER OTRO TIPO DE RE- } \\
\text { C U R O S PA R A L A } \\
\text { CONSTITUCIÓN, OPERACIÓN Y } \\
\text { ADMINISTRACION DE SOCIE- } \\
\text { DADES MERCANTILES, O }\end{array}$ & SIEMPRE & $\begin{array}{l}\text { CUANDO REA- } \\
\text { LICE OPERA- } \\
\text { CIONES A } \\
\text { NOMBRE DEL } \\
\text { CLIENTE }\end{array}$ \\
\hline & $\begin{array}{l}\text { E) LA CONSTITUCIÓN, } \\
\text { ESCISIÓN, FUSIÓN, OPERACIÓN } \\
\text { Y ADMINISTRACIÓN DE PER- } \\
\text { SONA S MOR A L E S O } \\
\text { VEHIICULOS CORPORATIVOS, } \\
\text { INCLUIDO EL FIDEICOMISO Y } \\
\text { LA COMPRA O VENTA DE ENTI- } \\
\text { DADES MERCANTILES. }\end{array}$ & SIEMPRE & $\begin{array}{l}\text { CUANDO REA- } \\
\text { LICE OPERA- } \\
\text { CIONES A } \\
\text { NOMBRE DEL } \\
\text { CLIENTE }\end{array}$ \\
\hline \multirow[b]{3}{*}{ F. XII } & \multicolumn{3}{|c|}{$\begin{array}{l}\text { LA PRESTACIÓN DE SERVICIOS DE FE PÚBLICA, EN LOS SI- } \\
\text { GUIENTES TÉRMINOS: }\end{array}$} \\
\hline & $\begin{array}{l}\text { APARTADO A. TRATÁNDOSE } \\
\text { DE LOS NOTARIOS PÚBLICOS: }\end{array}$ & & \\
\hline & $\begin{array}{l}\text { A) LA TRANSMISIÓN O } \\
\text { CONSTITUCIÓN DE DERECHOS } \\
\text { REALES SOBRE INMUEBLES, } \\
\text { SALVO LAS GARANTÍAS QUE } \\
\text { SE CONSTITUYAN EN FAVOR } \\
\text { DE INSTITUCIONES DEL SISTE- } \\
\text { MA FINANCIERO U ORGANIS- } \\
\text { MOS PÚBLICOS DE VIVIENDA. }\end{array}$ & SIEMPRE & 16,000 \\
\hline
\end{tabular}




\begin{tabular}{|c|c|c|c|}
\hline $\begin{array}{l}\text { FRACCIÓN DEL } \\
\text { ARTÍCULO } 17 \\
\text { DE LA LEY }\end{array}$ & ACTIVIDAD VULNERABLE & $\begin{array}{l}\text { UMBRAL DE } \\
\text { IDENTIFICA- } \\
\text { CIÓN EN UMAS }\end{array}$ & $\begin{array}{l}\text { UMBRAL DE } \\
\text { AVISO EN } \\
\text { UMAS }\end{array}$ \\
\hline \multirow{6}{*}{ F. XII } & \multicolumn{3}{|c|}{$\begin{array}{l}\text { LA PRESTACIÓN DE SERVICIOS DE FE PÚBLICA, EN LOS SI- } \\
\text { GUIENTES TÉRMINOS: }\end{array}$} \\
\hline & $\begin{array}{l}\text { APARTADO A. TRATÁNDOSE } \\
\text { DE LOS NOTARIOS PÚBLICOS: }\end{array}$ & & \\
\hline & $\begin{array}{l}\text { B) EL OTORGAMIENTO DE PO- } \\
\text { DERES PARA ACTOS DE } \\
\text { ADMINISTRACIÓN O DOMINIO } \\
\text { OTORGADOS CON CARÁCTER } \\
\text { IRREVOCABLE. LAS OPERA- } \\
\text { CIONES PREVISTAS EN ESTE } \\
\text { INCISO SIEMPRE SERÁN OBJE- } \\
\text { TO DE AVISO; }\end{array}$ & SIEMPRE & SIEMPRE \\
\hline & $\begin{array}{l}\text { C) LA CONSTITUCIÓN DE PER- } \\
\text { SONA S MOR ALES, SU } \\
\text { MODIFICACIÓN PATRIMONIAL } \\
\text { DERIVADA DE AUMENTO O } \\
\text { DISMINUCIÓN DE CAPITAL SO- } \\
\text { CIAL, FUSIÓN O ESCISIÓN, ASII } \\
\text { COMO LA COMPRAVENTA DE } \\
\text { ACCIONES Y PARTES SOCIALES } \\
\text { DE TALES PERSONAS; }\end{array}$ & SIEMPRE & 8,025 \\
\hline & $\begin{array}{l}\text { D) LA CONSTITUCIÓN O } \\
\text { MODIFICACIÓN DE FIDEICOMI- } \\
\text { SOS TRASLATIVOS DE DOMINIO } \\
\text { O DE GARANTÍA SOBRE INMUE- } \\
\text { BLES, SALVO LOS QUE SE CONS- } \\
\text { TITUYAN PARA GARANTIZAR } \\
\text { ALGÚN CRÉDITO A FAVOR DE } \\
\text { INSTITUCIONES DEL SISTEMA } \\
\text { FINANCIERO U ORGANISMOS } \\
\text { PÚBLICOS DE VIVIENDA; }\end{array}$ & SIEMPRE & 8,025 \\
\hline & $\begin{array}{l}\text { E) EL OTORGAMIENTO DE } \\
\text { CONTRATOS DE MUTUO O } \\
\text { CRÉDITO, CON O SIN } \\
\text { GARANTÍA, EN LOS QUE EL } \\
\text { ACREEDOR NO FORME PARTE } \\
\text { DEL SISTEMA FINANCIERO O } \\
\text { NO SEA UN ORGANISMO } \\
\text { PÚBLICO DE VIVIENDA. } \\
\end{array}$ & SIEMPRE & SIEMPRE \\
\hline & $\begin{array}{l}\text { APARTADO B. TRATÁNDOSE } \\
\text { DE LOS CORREDORES } \\
\text { PÚBLICOS: }\end{array}$ & & \\
\hline F. XII & $\begin{array}{l}\text { A) LA REALIZACIÓN DE } \\
\text { AVALÚOS SOBRE BIENES CON } \\
\text { VALOR IGUAL O SUPERIOR AL } \\
\text { EQUIVALENTE A OCHO MIL } \\
\text { VEINTICINCO VECES EL SALA- } \\
\text { RIO MÍNIMO VIGENTE EN EL } \\
\text { DISTRITO FEDERAL; }\end{array}$ & 8,025 & SIEMPRE \\
\hline
\end{tabular}




\begin{tabular}{|c|c|c|c|}
\hline $\begin{array}{l}\text { FRACCIÓN DEL } \\
\text { ARTÍCULO } 17 \\
\text { DE LA LEY }\end{array}$ & ACTIVIDAD VULNERABLE & $\begin{array}{l}\text { UMBRAL DE } \\
\text { IDENTIFICA- } \\
\text { CIÓN EN UMAS }\end{array}$ & $\begin{array}{l}\text { UMBRAL DE } \\
\text { AVISO EN } \\
\text { UMAS }\end{array}$ \\
\hline \multirow{4}{*}{ F. XII } & $\begin{array}{l}\text { APARTADO } \text { B. TRATÁNDOSE } \\
\text { DE LOS CORREDORES } \\
\text { PÚBLICOS: }\end{array}$ & & \\
\hline & $\begin{array}{l}\text { B) A CONSTITUCIÓN DE PERSO- } \\
\text { NAS MORALES MERCANTILES, } \\
\text { SU MODIFICACIÓN PATRIMO- } \\
\text { NIAL DERIVADA DE AUMENTO } \\
\text { O DISMINUCIÓN DE CAPITAL } \\
\text { SOCIAL, FUSIÓN O ESCISIÓN, } \\
\text { ASÍ COMO LA COMPRAVENTA } \\
\text { DE ACCIONES Y PARTES SOCIA- } \\
\text { LES DE PERSONAS MORALES } \\
\text { MERCANTILES; }\end{array}$ & SIEMPRE & SIEMPRE \\
\hline & $\begin{array}{l}\text { C) LA CONSTITUCIÓN, } \\
\text { MODIFICACIÓN O CESION DE } \\
\text { DERECHOS DE FIDEICOMISO, } \\
\text { EN LOS QUE DE ACUERDO CON } \\
\text { LA LEGISLACIÓN APLICABLE } \\
\text { PUEDAN ACTUAR; }\end{array}$ & SIEMPRE & SIEMPRE \\
\hline & $\begin{array}{l}\text { D) EL OTORGAMIENTO DE } \\
\text { CONTRATOS DE MUTUO MER- } \\
\text { CANTIL O CRÉDITOS MERCAN- } \\
\text { TILES EN LOS QUE DE ACUER- } \\
\text { DO CON LA LEGISLACIONN } \\
\text { APLICABLE PUEDAN ACTUAR } \\
\text { Y EN LOS QUE EL ACREEDOR } \\
\text { NO FORME PARTE DEL SISTE- } \\
\text { MA FINANCIERO. }\end{array}$ & SIEMPRE & SIEMPRE \\
\hline F. XIII & $\begin{array}{l}\text { LA RECEPCIÓN DE DONATI- } \\
\text { VOS, POR PARTE DE LAS ASO- } \\
\text { CIACIONES Y SOCIEDADES SIN } \\
\text { FINES DE LUCRO. }\end{array}$ & 1,605 & 3,210 \\
\hline \multirow[t]{2}{*}{ F.XIV } & \multicolumn{3}{|c|}{$\begin{array}{l}\text { LA PRESTACIÓN DE SERVICIOS DE COMERCIO EXTERIOR } \\
\text { COMO AGENTE O APODERADO ADUANAL, MEDIANTE } \\
\text { AUTORIZACIÓN OTORGADA POR LA SECRETARÍA DE HACIEN- } \\
\text { DA Y CRÉDITO PÚBLICO, PARA PROMOVER POR CUENTA AJE- } \\
\text { NA, EL DESPACHO DE MERCANCÍAS, EN LOS DIFERENTES } \\
\text { REGÍMENES ADUANEROS PREVISTOS EN LA LEY ADUANERA, } \\
\text { DE LAS SIGUIENTES MERCANCÍAS: }\end{array}$} \\
\hline & $\begin{array}{l}\text { A) VEHÍCULOS TERRESTRES, } \\
\text { AÉREOS Y MARÍTIMOS, NUE- } \\
\text { VOS Y USADOS, CUALQUIERA } \\
\text { QUE SEA EL VALOR DE LOS } \\
\text { BIENES; }\end{array}$ & SIEMPRE & SIEMPRE \\
\hline
\end{tabular}




\begin{tabular}{|c|c|c|c|}
\hline $\begin{array}{l}\text { FRACCIÓN DEL } \\
\text { ARTÍCULO } 17 \\
\text { DE LA LEY }\end{array}$ & ACTIVIDAD VULNERABLE & $\begin{array}{l}\text { UMBRAL DE } \\
\text { IDENTIFICA- } \\
\text { CIÓN EN UMAS }\end{array}$ & $\begin{array}{l}\text { UMBRAL DE } \\
\text { AVISO EN } \\
\text { UMAS }\end{array}$ \\
\hline \multirow{5}{*}{ F.XIV } & $\begin{array}{l}\text { B) MÁQUINAS PARA JUEGOS } \\
\text { DE APUESTA Y SORTEOS, NUE- } \\
\text { VAS Y USADAS, CUALQUIERA } \\
\text { QUE SEA EL VALOR DE LOS } \\
\text { BIENES; }\end{array}$ & SIEMPRE & SIEMPRE \\
\hline & $\begin{array}{l}\text { C) EQUIPOS Y MATERIALES } \\
\text { PARA LA ELABORACIÓN DE } \\
\text { TARJETAS DE PAGO, CUAL- } \\
\text { QUIERA QUE SEA EL VALOR DE } \\
\text { LOS BIENES; }\end{array}$ & SIEMPRE & SIEMPRE \\
\hline & $\begin{array}{l}\text { D) JOYAS, RELOJES, PIEDRAS } \\
\text { PRECIOSAS Y METALES PRE- } \\
\text { CIOSOS, CUYO VALOR INDIVI- } \\
\text { DUAL SEA IGUAL O SUPERIOR } \\
\text { AL EQUIVALENTE A CUATRO- } \\
\text { CIENTAS OCHENTA Y CINCO } \\
\text { VECES EL SALARIO MÍNIMO } \\
\text { VIGENTE EN EL DISTRITO FE- } \\
\text { DERAL; }\end{array}$ & 485 & SIEMPRE \\
\hline & $\begin{array}{l}\text { E) OBRAS DE ARTE, CUYO VA- } \\
\text { LOR INDIVIDUAL SEA IGUAL O } \\
\text { SUPERIOR AL EQUIVALENTE A } \\
\text { CUATRO MIL OCHOCIENTAS } \\
\text { QUINCE VECES EL SALARIO } \\
\text { MÍNIMO VIGENTE EN EL DIS- } \\
\text { TRITO FEDERAL; }\end{array}$ & 4,815 & SIEMPRE \\
\hline & $\begin{array}{l}\text { F) MATERIALES DE RESISTEN- } \\
\text { CIA BALÍSTICA PARA LA } \\
\text { PRESTACIÓN DE SERVICIOS DE } \\
\text { BLINDAJE DE VEHÍCULOS, } \\
\text { CUALQUIERA QUE SEA EL VA- } \\
\text { LOR DE LOS BIENES. }\end{array}$ & SIEMPRE & SIEMPRE \\
\hline F. XV & $\begin{array}{l}\text { LA CONSTITUCIÓN DE DERE- } \\
\text { CHOS PERSONALES DE USO O } \\
\text { GOCE DE BIENES INMUEBLES. }\end{array}$ & 1,605 & 3,210 \\
\hline F. XVI & $\begin{array}{l}\text { OFRECIMIENTO HABITUAL Y } \\
\text { PROFESIONAL DE INTERCAM- } \\
\text { BIO DE ACTIVOS VIRTUALES. }\end{array}$ & SIEMPRE & 645 \\
\hline
\end{tabular}

Adicional a las anteriores obligaciones, uno de los principales objetivos de la LFPIORPI es proteger el sistema financiero y la economía nacional, por ello, en términos del artículo 32 de la ley se establecen restricciones para el uso de efectivo en diversas operaciones.

Todas estas obligaciones y las demás que derivan de la LFPIOPI, RCG y el Reglamento de la misma, deberán estar contenidas en un documento que de manera interna elaboren los sujetos obligados, para definir los lineamientos, las 
medidas y los procedimientos para prevenir y detectar actos u operaciones que pudieran involucrar recursos de procedencia ilícita, que comúnmente se le conoce como Manual de Prevención de Lavado de Dinero.

\section{CONCLUSIÓN}

Las reformas a la LFPIORPI permitirán por una parte, cumplir con los requerimientos de GAFI hechos a México en el IEM 2018 con el propósito de fortalecer el sistema de prevención de lavado de dinero y financiamiento al terrorismo de las Actividades Vulnerables y por otra parte, definirán de manera más clara el contexto de la materia, por lo que considero relevante la aprobación de las mismas por parte del Senado, y de adoptar dichas reformas vendrían retos interesantes para los sujetos obligados puesto se deberá replantear el contexto de PLD/FT para visualizarlo desde un Enfoque Basado en Riesgo (EBR) y mediante la implementación de una metodología de EBR que califique los riesgos relacionados con las actividades que dichos sujetos obligados realicen con sus clientes.

Así también se vuelve relevante la implementación de sistemas automatizados de tecnologías efectivos, principalmente diseñados con el objetivo de facilitar los controles internos operativos con metodologías de EBR que permitan a quienes llevan a cabo Actividades Vulnerables, la toma de decisiones efectivas para evitar ser utilizados como medio para la realización de operaciones con recursos de procedencia ilícita y minimizar esos riesgos.

La Unidad de Inteligencia Financiera ha sido la autoridad encargada de darle seguimiento a dichas reformas y ha solicitado de manera reiterada su aprobación en diversos foros y medios.

La prevención de lavado de dinero debe tomarse en serio toda vez que es una línea importante del combate a la utilización de recursos de procedencia ilícita que debilita el sistema financiero y la economía nacional, y que constantemente se va actualizando porque los delincuentes utilizan nuevas tendencias para continuar celebrando todo tipo de operaciones ilícitas que les producen ganancias importantes, por lo que la participación de las Actividades Vulnerables en el marco de la ley resulta por demás relevante para frenar el paso de recursos de procedencia ilícita en el mercado nacional e internacional.

\section{ABREVIATURAS}

\begin{tabular}{|l|l|}
\hline Actividades Vulnerables & $\begin{array}{l}\text { A las actividades descritas en el artículo 17 de la Ley } \\
\text { Federal para la Prevención e Identificación de Opera- } \\
\text { ciones con Recursos de Procedencia Ilícita. }\end{array}$ \\
\hline ALA/CFT & $\begin{array}{l}\text { Antilavado de Activos y Contra Financiamiento al } \\
\text { Terrorismo. }\end{array}$ \\
\hline APNFD's & Actividades Profesionales No Financieras Designadas. \\
\hline
\end{tabular}




\begin{tabular}{|l|l|}
\hline CNBV & Comisión Nacional Bancaria y de Valores. \\
\hline GAFI & Grupo de Acción Financiera. \\
\hline IEM 2008 & $\begin{array}{l}\text { Informe de Evaluación Mutua realizada a México, } \\
\text { emitido por GAFI en el año 2008. }\end{array}$ \\
\hline IEM 2018 & $\begin{array}{l}\text { Informe de Evaluación Mutua realizada a México, } \\
\text { emitida por GAFI en el año 2018. }\end{array}$ \\
\hline LD/FT & Lavado de Dinero y Financiamiento al Terrorismo. \\
\hline LFPIORPI o la Ley & $\begin{array}{l}\text { Ley Federal para la Prevención e Identificación de } \\
\text { Operaciones con Recursos de Procedencia Ilícita. }\end{array}$ \\
\hline PLD/FT & $\begin{array}{l}\text { Prevención del Lavado de Dinero y Financiamiento } \\
\text { al Terrorismo. }\end{array}$ \\
\hline RCG & Reglas de Carácter General. \\
\hline RFC & Registro Federal de Contribuyentes. \\
\hline SAT & Servicio de Administración Tributaria. \\
\hline UMA & Unidades de Medidas y Actualización. \\
\hline
\end{tabular}

BIBLIOGRAFÍA

GONZÁLEZ RODRÍGUEZ, José de Jesús. "El lavado de dinero en México, escenarios, marco legal y propuestas legislativas". Documento de Trabajo número 66, abril de 2009. Centro de Estudios Sociales y de Opinión Publica.

Grupo de Acción Financiera. Estándares internacionales sobre la lucha contra el lavado de activos y el financiamiento del terrorismo y la proliferación. Las Recomendaciones de GAFI. Febrero de 2012.

Grupo de Acción Financiera. Mandato 2012-2020, abril 20, 2020, en Washington D.C.

Grupo de Acción Financiera. Medidas anti lavado y contra la financiación del terrorismo. México, Informe de Evaluación Mutua, 2018

Grupo de Acción Financiera. Metodología para evaluar el cumplimiento técnico con las recomendaciones del GAFI y la efectividad de los sistemas ALA/CFT. Febrero 2013.

\section{Legislación}

Acuerdo 02/2013 por el que se emiten Reglas de Carácter General a que se refiere la Ley Federal para la Prevención e Identificación de Operaciones con Recursos de Procedencia Ilícita. 2014.

Ley Federal para la Prevención e Identificación de Operaciones con Recursos de

Procedencia Ilícita. 2012. 
https://www.gob.mx/cms/uploads/attachment/file/80948/VSPP_GAFI 13042016.pdf

https://www.pld.hacienda.gob.mx/work/models/PLD/documentos/metodologia gafi.pdf

https://www.inegi.org.mx/temas/uma/\#: :text=La\%20Unidad\%20de\%20Medida $\% 20 y$,emanen $\% 20 \mathrm{de} \% 20$ todas $\% 201 \mathrm{las} \% 20$ anteriores

https://www.fatf-gafi.org/media/fatf/documents/reports/mer4/IEM-Mexico2018-Spanish.pdf 